\title{
The Annealed Entropy of Wiener Number on Random Double Hexagonal Chains
}

\author{
Haizhen Ren, Xueli Su \\ Department of Mathematics, Qinghai Normal University, Xining, China \\ Email: haizhenr@126.com
}

How to cite this paper: Ren, H.Z. and Su, X.L. (2017) The Annealed Entropy of Wiener Number on Random Double Hexagonal Chains. Applied Mathematics, 8, 1473-1480.

https://doi.org/10.4236/am.2017.810108

Received: September 7, 2017

Accepted: October 28, 2017

Published: October 31, 2017

Copyright @ 2017 by authors and Scientific Research Publishing Inc. This work is licensed under the Creative Commons Attribution International License (CC BY 4.0).

http://creativecommons.org/licenses/by/4.0/

\begin{abstract}
We study a random planar honeycomb lattice model, namely the random double hexagonal chains. This is a lattice system with nonperiodic boundary condition. The Wiener number is an important molecular descriptor based on the distances, which was introduced by the chemist Harold Wiener in 1947. By applying probabilistic method and combinatorial techniques we obtained an explicit analytical expression for the expected value of Wiener number of a random double hexagonal chain, and the limiting behaviors on the annealed entropy of Wiener number when the random double hexagonal chain becomes infinite in length are analyzed.
\end{abstract}

\section{Keywords}

Random Benzenoid Chain, Wiener Number, Entropy

\section{Introduction}

Topological indices (molecular structure descriptors) based on the distances between the vertices of a graph are widely used in theoretical chemistry to establish relations between the structure and the properties of molecules and provide correlations with physical, chemical, and thermodynamic parameters of chemical compounds [1]. Among the variety of these indices, the Wiener number, denoted by $W(G)$, is the best known one which was introduced by the chemist Harold Wiener in 1947 [2] as a simple parameter. Wiener number has been found to correlate with various physicochemical properties of a molecule (modeled by a graph): Boiling point, heat of vaporization, heat of isomerization, surface energy, specific dispersion, and sound velocity. In addition, the parameter also correlates with some $\pi$-electron characteristics of conjugated polymers; for example, the total $\pi$-electron energy and HOMO-LUMO (Highest 
Occupied Molecular Orbital-Lowest Unoccupied Molecular Orbital) separation, for details see [3] [4] and the references therein.

Let $G$ be a connected graph with vertices labeled as $1,2, \cdots, n$. The standard distance between two vertices $i$ and $j$, denoted by $d_{G}(i, j)$, is the length of a shortest path connecting them. In terms of graph theory, Wiener number [2] [3] is defined as the sum of distances between all pairs of vertices in $G$, i.e.,

$$
W(G)=\sum_{i<j} d_{G}(i, j)=\frac{1}{2} \sum_{i \in V(G)} \sum_{j \in V(G)} d_{G}(i, j)=\frac{1}{2} \sum_{i \in V(G)} d(i \mid G),
$$

where $d(i \mid G)$ is the Wiener number of vertex $i$ in $G$, defined by $d(i \mid G)=\sum d(i, j)$.

A hexagonali system is a 2-connected plane graph whose every interior face is bounded by a regular hexagon of unit length 1 . Hexagonal systems are of great importance for theoretical chemistry because they are the natural graph representation of benzenoid hydrocarbons [5] [6] [7] [8]. A hexagonal system $H$ is said to be catacondensed if all its vertices are on the outerface, otherwise $H$ is said to be pericondensed. In [4], Gutman et al. obtained an explicit analytical expression for the expected value of the Wiener number of a random benzenoid chain with $n$ hexagons(a graph of unbranched catacondensed benzenoid-like structure). The random multiple chain was introduced in [9], the generating procedure of which is inspired by the growth of single walled zigzag nanotubes [10]. Some results on double hexagonal chains (a special type of pericondensed hexagonal system with nonperiodic boundary condition, which is constructed by successively fusing a series of naphthalenes), can be found, for example, in [11] [12] [13] [14] [15] and the references therein. In statistical mechanics, entropy is related to the number of microscopic configurations that a thermodynamic system can have when in a state as specified by some macroscopic variables. In this paper, we study the annealed entropy of Wiener number on random double hexagonal chains.

The random double hexagonal chain $R D_{2 \times n}$ can be obtained from a naphthalene by stepwise triple-edge fusion of a new naphthalene. For convenience, we orient each naphthalene so that its interior edges are horizontal. There are two types of triple-edge fusion of two naphthalenes: $\alpha$-type fusion and $\beta$-type fusion, as shown in Figure 1(a). At each step $k(k \geq 3)$, a random selection is taken from one of the two possible fusions: $\alpha$-type fusion with probability $p$ and $\beta$-type fusion with probability $1-p$. In our model, we assume that the probability $p$ is a constant, invariant to the step parameter $k$. That is, the process described is a zeroth-order Markov process. For $n \geq 2, R D_{2 \times n}$ is a pericondensed hexagonal system. Random (double) hexagonal chains offer a good model for a class of conjugated polymers [16], many features of which have already been established [4] [11] [16]. By applying probabilistic method and combinatorial techniques an explicit analytical expression for the expected value $\mathbb{W}_{n}\left(=\mathbb{E}\left(W\left(R D_{2 \times n}\right)\right)\right)$ of the Wiener number of a random double hexagonal chain with $n$ naphthalenes is obtained. We note that the expression of $\mathbb{W}_{n}$ is a 


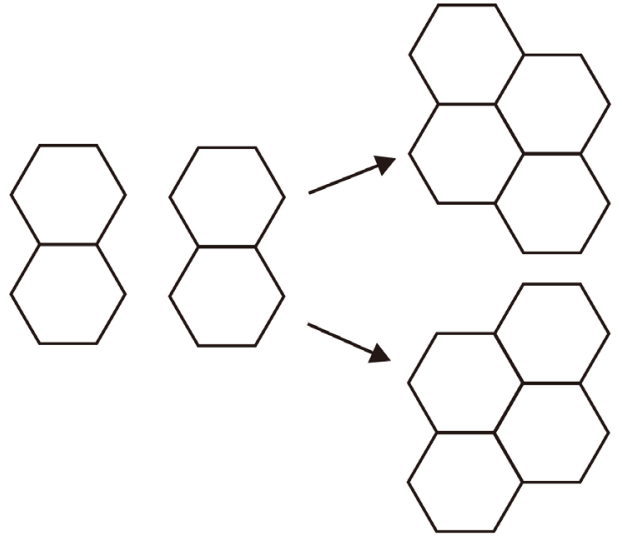

(a)
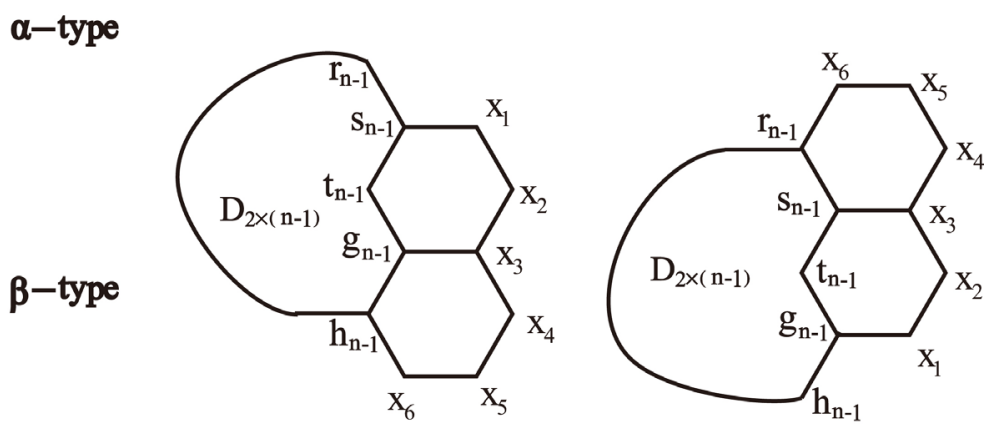

(b)

Figure 1. The triple-edge fusions. (a) $\alpha$-type fusion and $\beta$-type fusion. (b) $D_{2 \times n}$ is obtained by two possible fusions.

polynomial in the variable $n$. Furthermore, the limiting behaviors on the annealed entropy of Wiener number when the random double hexagonal chain becomes infinite in length are analyzed.

\section{Some Fundamental Recursion Relations}

A double hexagonal chain $D_{2 \times n}$ with $n$ naphthalenes can be constructed from $D_{2 \times(n-1)}$ by attaching a new terminal naphthalene spanned by vertices $\left\{s_{n-1}, t_{n-1}, g_{n-1}, h_{n-1}, x_{6}, x_{5}, x_{4}, x_{3}, x_{2}, x_{1}\right\}$ (or $\left\{r_{n-1}, s_{n-1}, t_{n-1}, g_{n-1}, x_{1}, x_{2}, x_{3}, x_{4}, x_{5}, x_{6}\right\}$ ) (see Figure 1(b)).

Lemma 2.1. If $D_{2 \times n}$ is obtained from $D_{2 \times(n-1)}$ by $\alpha$-type fusion, then

$$
\begin{aligned}
& d\left(x_{1} \mid D_{2 \times n}\right)=6 n+13+d\left(s_{n-1} \mid D_{2 \times(n-1)}\right), d\left(x_{2} \mid D_{2 \times n}\right)=12 n+1+d\left(s_{n-1} \mid D_{2 \times(n-1)}\right), \\
& d\left(x_{3} \mid D_{2 \times n}\right)=6 n+7+d\left(g_{n-1} \mid D_{2 \times(n-1)}\right), d\left(x_{4} \mid D_{2 \times n}\right)=12 n+5+d\left(g_{n-1} \mid D_{2 \times(n-1)}\right), \\
& d\left(x_{5} \mid D_{2 \times n}\right)=12 n+7+d\left(h_{n-1} \mid D_{2 \times(n-1)}\right), d\left(x_{6} \mid D_{2 \times n}\right)=6 n+13+d\left(h_{n-1} \mid D_{2 \times(n-1)}\right), \\
& \text { and } \\
& W\left(D_{2 \times n}\right)=W\left(D_{2 \times(n-1)}\right)+2\left[d\left(s_{n-1} \mid D_{2 \times(n-1)}\right)+d\left(g_{n-1} \mid D_{2 \times(n-1)}\right)\right. \\
& \left.+d\left(h_{n-1} \mid D_{2 \times(n-1)}\right)\right]+54 n+11 .
\end{aligned}
$$

Similarly, if $D_{2 \times n}$ is obtained from $D_{2 \times(n-1)}$ by $\beta$-type fusion, then

$$
\begin{aligned}
& d\left(x_{1} \mid D_{2 \times n}\right)=6 n+13+d\left(g_{n-1} \mid D_{2 \times(n-1)}\right), d\left(x_{2} \mid D_{2 \times n}\right)=12 n+1+d\left(g_{n-1} \mid D_{2 \times(n-1)}\right), \\
& d\left(x_{3} \mid D_{2 \times n}\right)=6 n+7+d\left(s_{n-1} \mid D_{2 \times(n-1)}\right), d\left(x_{4} \mid D_{2 \times n}\right)=12 n+5+d\left(s_{n-1} \mid D_{2 \times(n-1)}\right), \\
& d\left(x_{5} \mid D_{2 \times n}\right)=12 n+7+d\left(r_{n-1} \mid D_{2 \times(n-1)}\right), d\left(x_{6} \mid D_{2 \times n}\right)=6 n+13+d\left(r_{n-1} \mid D_{2 \times(n-1)}\right), \\
& \text { and } \\
& W\left(D_{2 \times n}\right)=W\left(D_{2 \times(n-1)}\right)+2\left[d\left(s_{n-1} \mid D_{2 \times(n-1)}\right)+d\left(g_{n-1} \mid D_{2 \times(n-1)}\right)\right. \\
& \left.+d\left(r_{n-1} \mid D_{2 \times(n-1)}\right)\right]+54 n+11, \\
& \text { where } d\left(s_{1} \mid D_{2 \times 1}\right)=d\left(g_{1} \mid D_{2 \times 1}\right)=21, d\left(h_{1} \mid D_{2 \times 1}\right)=d\left(r_{1} \mid D_{2 \times 1}\right)=25 \text {. }
\end{aligned}
$$


Proof. By (1), we have $d\left(s_{1} \mid D_{2 \times 1}\right)=d\left(g_{1} \mid D_{2 \times 1}\right)=21$, $d\left(h_{1} \mid D_{2 \times 1}\right)=d\left(r_{1} \mid D_{2 \times 1}\right)=25$. Since a naphthalene is not a vertex rotation symmetry, we must distinguish between two different situations for the Wiener number of the vertex in the terminal naphthalene. In the following, we only consider the case of $\alpha$-type fusion (the argument for the case of $\beta$-type fusion is analogous). Note that $D_{2 \times(n-1)}$ has $6 n-2$ vertices. If $D_{2 \times n}$ is obtained from $D_{2 \times(n-1)}$ by $\alpha$-type fusion then, for $n \geq 2$, we have the following relations:

$$
\begin{aligned}
& d\left(x_{1} \mid D_{2 \times n}\right)=6 n-2+d\left(s_{n-1} \mid D_{2 \times(n-1)}\right)+15, \\
& d\left(x_{2} \mid D_{2 \times n}\right)=2(6 n-2)+\Delta_{n-1}-6+11 \text {, } \\
& d\left(x_{3} \mid D_{2 \times n}\right)=6 n-2+d\left(g_{n-1} \mid D_{2 \times(n-1)}\right)+9, \\
& d\left(x_{4} \mid D_{2 \times n}\right)=2(6 n-2)+d\left(g_{n-1} \mid D_{2 \times(n-1)}\right)+9 \text {, } \\
& d\left(x_{5} \mid D_{2 \times n}\right)=2(6 n-2)+d\left(h_{n-1} \mid D_{2 \times(n-1)}\right)+11, \\
& d\left(x_{6} \mid D_{2 \times n}\right)=6 n-2+d\left(h_{n-1} \mid D_{2 \times(n-1)}\right)+15 \text {. } \\
& \text { and } \\
& W\left(D_{2 \times n}\right)=W\left(D_{2 \times(n-1)}\right)+d\left(s_{n-1} \mid D_{2 \times(n-1)}\right)+2\left[d\left(g_{n-1} \mid D_{2 \times(n-1)}\right)\right. \\
& \left.+d\left(h_{n-1} \mid D_{2 \times(n-1)}\right)\right]+\Delta_{n-1}+54 n+11,
\end{aligned}
$$

where $\Delta_{n-1}=\min \left\{d\left(s_{n-1} \mid D_{2 \times(n-1)}\right), d\left(g_{n-1} \mid D_{2 \times(n-1)}\right)\right\}$. In this case, $s_{n-1}$ and $g_{n-1}$ coincide with the vertices $x_{2}$ and $x_{4}$, respectively. Thus,

$d\left(s_{n-1} \mid D_{2 \times(n-1)}\right)-d\left(g_{n-1} \mid D_{2 \times(n-1)}\right)=\Delta_{n-2}-4-d\left(g_{n-2} \mid D_{2 \times(n-1)}\right)<0$. By induction on $n$, we know that $\Delta_{n-1}=d\left(s_{n-1} \mid D_{2 \times(n-1)}\right)$. The proof is completed.

For a random double benzenoid chain $R D_{2 \times n}$, there are two cases to be considered:

Case 1. $R D_{2 \times n} \rightarrow R D_{2 \times(n+1)}$ by $\alpha$-type fusion with probability $p$. In this case, $r_{n}, s_{n}, t_{n}, g_{n}, h_{n}$ (of $R D_{2 \times n}$ ) coincide with the vertices $x_{1}, x_{2}, x_{3}, x_{4}, x_{5}$, respectively.

Case 2. $R D_{2 \times n} \rightarrow R D_{2 \times(n+1)}$ by $\beta$-type fusion with probability $1-p$. In this case, $r_{n}, s_{n}, t_{n}, g_{n}, h_{n}$ (of $R D_{2 \times n}$ ) coincide with the vertices $x_{5}, x_{4}, x_{3}, x_{2}, x_{1}$, respectively.

The distance $d\left(r_{n} \mid R D_{2 \times n}\right), d\left(s_{n} \mid R D_{2 \times n}\right), d\left(g_{n} \mid R D_{2 \times n}\right), d\left(h_{n} \mid R D_{2 \times n}\right)$ and $W\left(R D_{2 \times(n-1)}\right)$ are random variables. We denote their expected values by $\mathbb{R}_{n}=\mathbb{E}\left(d\left(r_{n} \mid R D_{2 \times n}\right)\right), \quad \mathbb{S}_{n}=\mathbb{E}\left(d\left(s_{n} \mid R D_{2 \times n}\right)\right), \quad \mathbb{G}_{n}=\mathbb{E}\left(d\left(g_{n} \mid R D_{2 \times n}\right)\right)$, $\mathbb{H}_{n}=\mathbb{E}\left(d\left(h_{n} \mid R D_{2 \times n}\right)\right)$ and $\mathbb{W}_{n}=\mathbb{E}\left(W\left(R D_{2 \times n}\right)\right) \quad$ (or $E(W(n))$ in brief), respectively. Then, by Case 1, Case 2 and Lemma 2.1 we have

$$
\begin{aligned}
& \mathbb{R}_{n}=p\left(6 n+13+d\left(s_{n-1} \mid R D_{2 \times(n-1)}\right)\right)+(1-p)\left(12 n+7+d\left(r_{n-1} \mid R D_{2 \times(n-1)}\right)\right) ; \\
& \mathbb{S}_{n}=p\left(12 n+1+d\left(s_{n-1} \mid R D_{2 \times(n-1)}\right)\right)+(1-p)\left(12 n+5+d\left(s_{n-1} \mid R D_{2 \times(n-1)}\right)\right) ; \\
& \mathbb{G}_{n}=p\left(12 n+5+d\left(g_{n-1} \mid R D_{2 \times(n-1)}\right)\right)+(1-p)\left(12 n+1+d\left(g_{n-1} \mid R D_{2 \times(n-1)}\right)\right) ; \\
& \mathbb{H}_{n}=p\left(12 n+7+d\left(h_{n-1} \mid R D_{2 \times(n-1)}\right)\right)+(1-p)\left(6 n+13+d\left(g_{n-1} \mid R D_{2 \times(n-1)}\right)\right)
\end{aligned}
$$


and

$$
\begin{aligned}
\mathbb{W}_{n}= & W\left(R D_{2 \times(n-1)}\right)+2\left(d\left(s_{n-1} \mid R D_{2 \times(n-1)}\right)+d\left(g_{n-1} \mid R D_{2 \times(n-1)}\right)\right) \\
& +2 p d\left(h_{n-1} \mid R D_{2 \times(n-1)}\right)+2(1-p) d\left(r_{n-1} \mid R D_{2 \times(n-1)}\right)+54 n+11 .
\end{aligned}
$$

Theorem 2.1. For $n \geq 2$, then

$$
\begin{aligned}
& \mathbb{R}_{n}=p\left(6 n+13+\mathbb{S}_{n-1}\right)+(1-p)\left(12 n+7+\mathbb{R}_{n-1}\right) \\
& \mathbb{S}_{n}=12 n+5-4 p+\mathbb{S}_{n-1} ; \\
& \mathbb{G}_{n}=12 n+1+4 p+\mathbb{G}_{n-1} ; \\
& \mathbb{H}_{n}=p\left(12 n+7+\mathbb{H}_{n-1}\right)+(1-p)\left(6 n+13+\mathbb{G}_{n-1}\right) \\
& \mathbb{W}_{n}=\mathbb{W}_{n-1}+2\left(\mathbb{S}_{n-1}+\mathbb{G}_{n-1}\right)+2 p \mathbb{H}_{n-1}+2(1-p) \mathbb{R}_{n-1}+54 n+11
\end{aligned}
$$

with boundary conditions $\mathbb{W}_{1}=109, \mathbb{S}_{1}=\mathbb{G}_{1}=21$ and $\mathbb{R}_{1}=\mathbb{H}_{1}=25$.

Proof. Noting that $\mathbb{E}\left(\mathbb{R}_{n}\right)=\mathbb{R}_{n}, \mathbb{E}\left(\mathbb{S}_{n}\right)=\mathbb{S}_{n}, \mathbb{E}\left(\mathbb{G}_{n}\right)=\mathbb{G}_{n}, \mathbb{E}\left(\mathbb{H}_{n}\right)=\mathbb{H}_{n}$ and $\mathbb{E}\left(\mathbb{W}_{n}\right)=\mathbb{W}_{n}$. Thus, Theorem 2.1 holds.

\section{The Explicit Analytical Expression for $\mathbb{W}_{n}$}

From (2), by successive subtraction method we have

$$
\mathbb{S}_{n}=6 n^{2}+11 n-4 p n+4 p+4, \mathbb{G}_{n}=6 n^{2}+7 n+4 p n-4 p+8 .
$$

Therefore,

$$
\begin{aligned}
& \mathbb{R}_{n}=12 n+5 p-7 n p+6 n^{2} p+8 p^{2}-4 n p^{2}+7+(1-p) \mathbb{R}_{n-1}, \\
& \mathbb{H}_{n}=20+n+6 n^{2}-21 p+15 n p-6 n^{2} p+8 p^{2}-4 n p^{2}+p \mathbb{H}_{n-1}, \\
& \mathbb{W}_{n}=\mathbb{W}_{n-1}+2 p \mathbb{H}_{n-1}+2(1-p) \mathbb{R}_{n-1}+23+42 n+24 n^{2} .
\end{aligned}
$$

Thus, we get the following result.

Theorem 3.1. For $n \geq 2$, if $p=0$ then $\mathbb{R}_{n}=6 n^{2}+13 n+6, \mathbb{S}_{n}=6 n^{2}+11 n$ $+4, \mathbb{G}_{n}=6 n^{2}+7 n+8, \mathbb{H}_{n}=20+n+6 n^{2}, \mathbb{W}_{n}=12 n^{3}+40 n^{2}+49 n+8 ;$ and if $p=1$ then $\mathbb{R}_{n}=6 n^{2}+n+20, \mathbb{S}_{n}=6 n^{2}+7 n+8, \quad \mathbb{G}_{n}=6 n^{2}+11 n+4$, $\mathbb{H}_{n}=6 n^{2}+13 n+6, \quad \mathbb{W}_{n}=12 n^{3}+40 n^{2}+49 n+8$.

We now consider the case when $0<p<1$ by using the method of generating functions. Let

$$
\mathbb{R}(t)=\sum_{n \geq 0} \mathbb{R}_{n} t^{n}, \mathbb{H}(t)=\sum_{n \geq 0} \mathbb{H}_{n} t^{n}, \mathbb{W}(t)=\sum_{n \geq 0} \mathbb{W}_{n} t^{n}
$$

Then, by (4) we get

$$
\begin{aligned}
& \mathbb{R}(t)-\mathbb{R}_{0}=(1-p) t \mathbb{R}(t)+\left(19+4 p+4 p^{2}\right) \delta_{3}+\left(12+5 p-4 p^{2}\right) \delta_{2}+6 p \delta_{1}, \\
& \mathbb{H}(t)-\mathbb{H}_{0}=p t \mathbb{H}(t)+\left(27-12 p+4 p^{2}\right) \delta_{3}+\left(13+3 p-4 p^{2}\right) \delta_{2}+(6-6 p) \delta_{1}, \\
& \mathbb{W}(t)-\mathbb{W}_{0}=t \mathbb{W}(t)+2 p t \mathbb{H}(t)+2(1-p) t \mathbb{R}(t)+89 \delta_{3}+90 \delta_{2}+24 \delta_{1},
\end{aligned}
$$

where $\delta_{1}=\sum_{n \geq 0} n^{2} t^{n+1}, \delta_{2}=\sum_{n \geq 0} n t^{n+1}$ and $\delta_{3}=\sum_{n \geq 0} t^{n+1}$. Since $(1-t)^{-j}=\sum_{n \geq 0} C_{n}^{j-1} t^{n-j+1}$.

Then $(1-p t)^{-1}=\sum_{n \geq 0}(p t)^{n}$ and $(1-t)^{-1}=\sum_{n \geq 0} t^{n}$. Thus, by (5) we have 


$$
\begin{aligned}
\mathbb{R}(t)= & \sum_{n \geq 0}((1-p) t)^{n}\left[\mathbb{R}_{0}+\left(19+4 p+4 p^{2}\right) \sum_{m \geq 1} t^{m}\right. \\
& \left.+\left(12+5 p-4 p^{2}\right) \sum_{m \geq 1}(m-1) t^{m}+6 p \sum_{m \geq 1}(m-1)^{2} t^{m}\right] \\
= & \sum_{n \geq 0} \mathbb{R}_{0}((1-p) t)^{n}+\sum_{k \geq 0} \sum_{n+m=k, m \geq 1}(1-p)^{n}\left[\left(19+4 p+4 p^{2}\right)\right. \\
& \left.+\left(12+5 p-4 p^{2}\right)(m-1)+6 p(m-1)^{2}\right] t^{k} \\
= & \sum_{n \geq 0} \mathbb{R}_{0}((1-p) t)^{n}+\sum_{n \geq 0} \sum_{m=1}^{n}(1-p)^{n-m}\left[\left(19+4 p+4 p^{2}\right)\right. \\
& \left.\left.+\left(12+5 p-4 p^{2}\right)(m-1)+6 p(m-1)^{2}\right]\right] t^{n} \\
= & \sum_{n \geq 0} \mathbb{R}_{n} t^{n},
\end{aligned}
$$

where,

$$
\begin{aligned}
& \mathbb{R}_{n}=\mathbb{R}_{0}(1-p)^{n}+\sum_{m=2}^{n}(1-p)^{n-m}\left[7+12 m+5 p-7 m p+6 m^{2} p+8 p^{2}-4 m p^{2}\right], \\
& \mathbb{H}(t)=\sum_{n \geq 0}(p t)^{n}\left(\mathbb{H}_{0}+\sum_{m \geq 1}\left[\left(27-12 p+4 p^{2}\right)\right.\right. \\
& \left.\left.+\left(13+3 p-4 p^{2}\right)(m-1)+(6-6 p)(m-1)^{2}\right] t^{m}\right) \\
& =\sum_{n \geq 0} \mathbb{H}_{0}(p t)^{n}+\sum_{k \geq 0} \sum_{n+m=k, m \geq 1} p^{n}\left(20+m+6 m^{2}-21 p+15 m p-6 m^{2} p+8 p^{2}-4 m p^{2}\right) t^{k} \\
& =\sum_{n \geq 0} \mathbb{H}_{0}(p t)^{n}+\sum_{n \geq 0} \sum_{m=1}^{n} p^{n-m}\left[20+m+6 m^{2}-21 p+15 m p-6 m^{2} p+8 p^{2}-4 m p^{2}\right] t^{n} \\
& =\sum_{n \geq 0} \mathbb{H}_{n} t^{n}, \\
& \mathbb{H}_{n}=\mathbb{H}_{0} p^{n}+\sum_{m=1}^{n} p^{n-m}\left[20+m+6 m^{2}-21 p+15 m p-6 m^{2} p+8 p^{2}-4 m p^{2}\right], \\
& \mathbb{W}(t)=\sum_{n \geq 0} t^{n}\left[\mathbb{W}_{0}+2 p \sum_{m \geq 1} \mathbb{H}_{m-1} t^{m}+2(1-p) \sum_{m \geq 1} \mathbb{R}_{m-1} t^{m}+\sum_{m \geq 1}\left(23+42 m+24 m^{2}\right) t^{m}\right] \\
& =\sum_{n \geq 0}\left[\mathbb{W}_{0}+\sum_{m=1}^{n}\left(2 p \mathbb{H}_{m-1}+2(1-p) \mathbb{R}_{m-1}+23+42 m+24 m^{2}\right) t^{n}\right] \\
& =\sum_{n \geq 0} \mathbb{W}_{n} t^{n}, \\
& \mathbb{W}_{n}=\mathbb{W}_{0}+\sum_{m=1}^{n}\left(2 p \mathbb{H}_{m-1}+2(1-p) \mathbb{R}_{m-1}+23+42 m+24 m^{2}\right) .
\end{aligned}
$$

Thus, we reach the following result.

Theorem 3.2. If $n \geq 2$ and $0<p<1$, then

$$
\begin{aligned}
& \mathbb{R}_{n}=\mathbb{R}_{0}(1-p)^{n}+\sum_{m=2}^{n}(1-p)^{n-m}\left[7+12 m+5 p-7 m p+6 m^{2} p+8 p^{2}-4 m p^{2}\right], \\
& \mathbb{S}_{n}=6 n^{2}+11 n-4 p n+4 p+4, \\
& \mathbb{G}_{n}=6 n^{2}+7 n+4 p n-4 p+8, \\
& \mathbb{H}_{n}=\mathbb{H}_{0} p^{n}+\sum_{m=1}^{n} p^{n-m}\left[20+m+6 m^{2}-21 p+15 m p-6 m^{2} p+8 p^{2}-4 m p^{2}\right], \\
& \mathbb{W}_{n}=\mathbb{W}_{0}+\sum_{m=1}^{n}\left(2 p \mathbb{H}_{m-1}+2(1-p) \mathbb{R}_{m-1}+23+42 m+24 m^{2}\right) .
\end{aligned}
$$


It is easy to verify that $\sum_{m=0}^{n} p^{n-m}=\frac{1-p^{n+1}}{1-p}, \sum_{m=1}^{n} m p^{n-m}=\frac{p^{n}-(n+1)}{p-1}+\frac{p^{n}-1}{(p-1)^{2}}$, $\sum_{m=1}^{n} m p^{m-1}=\frac{1-(n+1) p^{n}}{1-p}+\frac{p\left(1-p^{n}\right)}{(1-p)^{2}}$ and $\sum_{m=1}^{n} m^{2} p^{n-m}=\left(-n^{2}-p+2 n p+2 n^{2} p-p^{2}-2 n p^{2}-n^{2} p^{2}+p^{1+n}+p^{2+n}\right) /(-1+p)^{3}$.

So by (4), we can calculate the values of $\mathbb{R}_{0}, \mathbb{H}_{0}$ and $\mathbb{W}_{0}$, and by using Mathematica software(Mathematica 9.0) to (6) we obtain the solution of (6) as follows:

Theorem 3.3. For $n \geq 2$ and $0<p<1$, then

$$
\begin{gathered}
\mathbb{R}_{n}=\frac{1}{(1-p) p}\left(8-8(1-p)^{n}+5 n p+6 n^{2} p+6(1-p)^{n} p\right. \\
\left.-4 p^{2}-9 n p^{2}-6 n^{2} p^{2}-4 p^{3}+4 n p^{3}\right), \\
\mathbb{S}_{n}=6 n^{2}+11 n-4 p n+4 p+4, \\
\mathbb{G}_{n}=6 n^{2}+7 n+4 p n-4 p+8, \\
\mathbb{H}_{n}=\frac{1}{(-1+p) p}\left(-20 p-n p-6 n^{2} p+16 p^{2}-3 n p^{2}\right. \\
\left.+6 n^{2} p^{2}-4 p^{3}+4 n p^{3}+2 p^{n}+6 p^{1+n}\right), \\
\mathbb{W}_{n}=\frac{1}{(-1+p)^{2} p^{2}}\left(-16+16(1-p)^{n}+44 p+16 n p-44(1-p)^{n} p-32 p^{2}\right. \\
+13 n p^{2}+32 n^{2} p^{2}+12 n^{3} p^{2}+40(1-p)^{n} p^{2}-40 p^{3}-34 n p^{3}-72 n^{2} p^{3} \\
-24 n^{3} p^{3}-12(1-p)^{n} p^{3}+60 p^{4}-43 n p^{4}+56 n^{2} p^{4}+12 n^{3} p^{4}-48 p^{5} \\
\left.+72 n p^{5}-24 n^{2} p^{5}+16 p^{6}-24 n p^{6}+8 n^{2} p^{6}+4 p^{2+n}+12 p^{3+n}\right)
\end{gathered}
$$

\section{Conclusions and Suggestions}

From Theorem 3.1 and Theorem 3.3, we know that $\mathbb{W}_{n} \sim 12 n^{3}$ as $n$ tends to infinity. Note that $\mathbb{W}_{n}$ is a polynomial in the variable $n$, which is different from the expected value of the Wiener number of the random benzenoid chain in the general case. And the limiting behaviors on the annealed entropy $\log \mathbb{W}_{n}$ when the random double hexagonal chain becomes infinite in length are

$$
\lim _{m \rightarrow \infty} \frac{1}{V} \log \left[\mathbb{W}_{n}\right]=0,
$$

where $V$ is the number of the vertices in $R D_{2 \times n}$, i.e., $V=6 n+4$.

Open problem. For any $p \neq 0,1$ and $n \geq 1$, is it true that the annealed entropy $\log \mathbb{W}_{n}$ of $m$-tuple random hexagonal chain is zero as $m \rightarrow \infty$ or $m, n \rightarrow \infty$.

\section{Acknowledgements}

Sincere thanks to the members of JAMP for their professional performance, and 
special thanks to managing editor for a rare attitude of high quality. This research supported by NSFC(11551003) and the Natural Science Foundation of Qinghai(2015-ZJ-911).

\section{References}

[1] Gutman, I. and Furtula, B., Eds. (2012) Distance in Molecular Graphs-Theory (Distance in Molecular Graphs-Applications). University of Kragujevac, Kragujevac.

[2] Wiener, H. (1947) Structural Determination of Paraffin Boiling Points. Journal of the American Chemical Society, 69, 17-20. https://doi.org/10.1021/ja01193a005

[3] Dobrynin, A., Entringer, R. and Gutman, I. (2001) Wiener Index of Trees: Theory and Applications. Acta Applicandae Mathematicae, 66, 211-249. https://doi.org/10.1023/A:1010767517079

[4] Gutman, I., Kennedy, J.W. and Quintas, L.V. (1990) Wiener Numbers of Random Benzenoid Chains. Chemical Physics Letters, 173, 403-408. https://doi.org/10.1016/0009-2614(90)85292-K

[5] Gutman, I. and Polansky, O.E. (1986) Mathematical Concepts in Organic Chemistry. Springer, Berlin. https://doi.org/10.1007/978-3-642-70982-1

[6] Gutman, I. and Cyvin, S.J., Eds. (1989) Introduction to the Theory of Benzenoid Hydrocarbons. Springer, Berlin. https://doi.org/10.1007/978-3-642-87143-6

[7] Gutman, I. and Cyvin, S.J., Eds. (1990) Advances in the Theory of Benzenoid Hydrocarbons. Topics in Current Chemistry, Vol. 153, Springer, Berlin. https://doi.org/10.1007/3-540-51505-4

[8] Gutman, I., Ed. (1992) Advances in the Theory of Benzenoid Hydrocarbons I. Topics in Current Chemistry, Vol. 162, Springer, Berlin.

[9] Ren, H.Z., Zhang, F.J. and Qian, J.G. (2012) Dimer Coverings on Random Multiple Chains of Planar Honeycomb Lattices. Journal of Statistical Mechanics. Theory and Experiment, 2012, P08002. https://doi.org/10.1088/1742-5468/2012/08/P08002

[10] Dresselhaus, M.S., Dresselhaus, G. and Avouris, P. (2001) Carbon Nanotubes: Synthesis, Structure, Properties and Applications. Springer, Berlin. https://doi.org/10.1007/3-540-39947-X

[11] Gutman I., Randic, M., Balaban, A., Furtula, B. and Vuckovic, V. (2005) $\pi$-Electron Contents of Rings in the Double-Hexagonal-Chain Homologous Series (Pyrene, Anthanthrene and Other Acenoacenes). Polycyclic Aromatic Compounds, 25, 215-226. https://doi.org/10.1080/10406630591007080

[12] Ren, H.Z. and Zhang, F.J. (2007) Double Hexagonal Chains with Maximal Energy. International Journal of Quantum Chemistry, 107, 1437-1445. https://doi.org/10.1002/qua.21256

[13] Ren, H.Z. and Zhang, F.J. (2007) Double Hexagonal Chains with Minimal Total $\pi$-Electron Energy. Journal of Mathematical Chemistry, 42, 1041-1056. https://doi.org/10.1007/s10910-006-9159-9

[14] Ren, H.Z. and Zhang, F.J. (2007) Extremal Double Hexagonal Chains with Respectfully Yours, to k-Matchings and $k$-Independent Sets. Discrete Applied Mathematics, 155, 2269-2281. https://doi.org/10.1016/j.dam.2007.06.003

[15] Deng, H. (2008) The Anti-Forcing Number of Double Hexagonal Chains. MATCH Communications in Mathematical and in Computer Chemistry, 60, 183-192.

[16] Klein, D.J., Živković, T.P. and Trinajstić, N. (1987) Resonance in Random $\pi$-Network Polymers. Journal of Mathematical Chemistry, 1, 309-334. https://doi.org/10.1007/BF01179796 